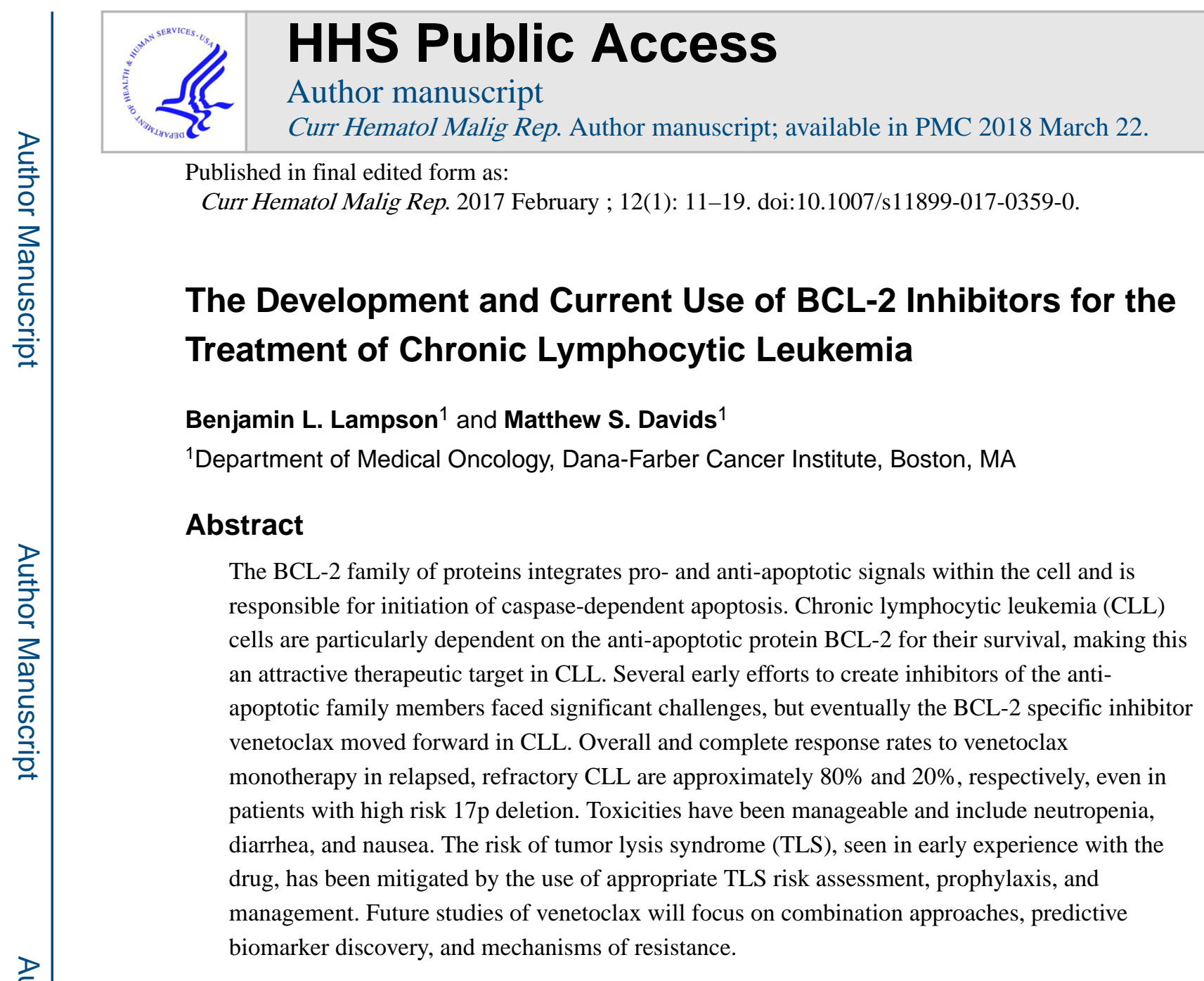

Keywords

Venetoclax; chronic lymphocytic leukemia; BCL-2 inhibitors; clinical trials

\title{
Introduction
}

The past decade has seen the emergence of a new class of cancer treatment that operates by directly promoting the cellular process of apoptosis, or programmed cell death. The development of BCL-2 inhibitors began with multiple unsuccessful candidates before finally arriving at venetoclax, which was recently FDA-approved. Here, we review the development of BCL-2 inhibitors and their emergence as a powerful class of agents for the treatment of chronic lymphocytic leukemia (CLL).

\section{Apoptosis and the BCL-2 Family}

Apoptosis is an orderly, programmed intracellular signaling cascade that leads to cell death. BCL-2 encodes an anti-apoptotic protein and was the first oncogene identified that primarily

Corresponding Author: Matthew S. Davids, MD, MMSc, Dana-Farber Cancer Institute, 450 Brookline Ave, Boston, MA 02215, matthew_davids@dfci.harvard.edu. Telephone: 617-632-5847. Fax: 617-582-7890.

Mailing Addresses: Benjamin L. Lampson, MD, PhD, Dana-Farber Cancer Institute, 450 Brookline Ave, Boston, MA 02215,

blampson@partners.org. Telephone: 617-632-3779. Fax: 617-632-5822. 
exerted its oncogenic effects by inhibiting cell death, rather than by promoting uncontrolled cellular proliferation. Subsequently, additional proteins have been identified that share one or more functional domains with BCL-2. The proteins in this BCL-2 family fall into three groups based on their structure and function (reviewed in [1]). Anti-apoptotic proteins, including BCL-2, MCL-1, BCL- $\mathrm{X}_{\mathrm{L}}$, BCL-w and others, have four tandem BCL-2 homology (BH) domains and ultimately promote cellular survival. Accordingly, their expression is upregulated downstream of pro-survival signaling pathways such as phosphatidylinositol-3kinase (PI3K) and AKT [2]. Pro-apoptotic BH3-only proteins, including BIM, PUMA, and $\mathrm{BAD}$, have a single $\mathrm{BH}$ domain, and promote cell death. They are also transcriptional targets of tumor suppressors such as TP53 [3]. The pro-apoptotic effector proteins BAX and BAK are required to carry out the pro-apoptotic effects mediated by other proteins within the family.

BCL-2 family proteins reside on the outer surface of the mitochondrial membrane and function through direct protein-protein interactions with each other, mediated by the $\mathrm{BH}$ domain. BH3-only proteins, when not sequestered by anti-apoptotic BCL-2-like family members, can promote the homo-oligomerization of the effector proteins BAX and BAK, leading to mitochondrial outer membrane permeabilization (MOMP), release of cytochrome c, and activation of the caspase cascade [4]. Thus, BCL-2 family members can be thought of as a group of intracellular sensors which detect the net pro- or anti-apoptotic state of the cell, and when the balance tips in favor of apoptosis, they ultimately initiate the irreversible step of MOMP.

This leads to some important conclusions with regard to the clinical relevance of targeting the BCL-2 pathway. First, one method to promote cell death would be to pharmacologically inhibit protein-protein interactions between pro-apoptotic and anti-apoptotic BCL-2 family members, thus freeing pro-apoptotic BH3-only proteins to promote MOMP. Second, directly targeting BCL-2 family members bypasses the need for an intact TP53 signaling axis, as TP53 lies upstream of these proteins.

Functional analysis of a cell's proximity to the apoptotic threshold (known as "mitochondrial priming") can be performed through an assay called BH3 profiling [5, 6]. This assay assesses the amount of carefully titrated BH3-only peptides which interact directly with mitochondria to trigger MOMP (defined as release of cytochrome c). BH3 profiling of CLL cells indicates that these cells are generally highly primed for apoptosis [7]. Supporting this finding, BCL-2 protein expression levels are typically higher in CLL cells than in peripheral blood mononuclear cells. Additionally, BCL-2 sequesters large amounts of the pro-apoptotic BH3-only protein BIM, causing CLL cells to be highly dependent on BCL-2 for survival [7]. Although CLL cells depend on BCL-2 for survival, they have not been found to harbor genetic mutations or translocations of BCL-2 family members. This mirrors the functional dependence of CLL cells on the B cell receptor (BCR) signaling pathway, where activating mutations of key proteins such as Bruton's Tyrosine Kinase (BTK) are also not present [8]. 


\section{Early Attempts at Targeting BCL-2 in the Clinic Oblimersen}

Oblimersen is an antisense oligodeoxynucleotide designed to target BCL-2 mRNA [9]. It was found to decrease BCL-2 expression and the proliferation rate of lymphoma cell lines [10]. A phase I/II study in relapsed/refractory CLL identified a cytokine release syndrome, consisting of fevers and hypotension, as the dose limiting toxicity. Efficacy as a single agent was modest, with only 2 of 26 evaluable patients (8\%) having a partial response [11]. The drug was later tested in a phase III trial, with patients randomized to fludarabine/ cyclophosphamide or fludarabine/cyclophosphamide/oblimersen. There was no significant difference in the 5-year survival rate between the two arms in an intent-to-treat analysis [12], and further development of this agent in CLL was halted.

\section{Gossypol Compounds}

Gossypol is a polyphenolic aldehyde isolated from the cotton plant. Gossypol directly interacts with BCL- $\mathrm{X}_{\mathrm{L}}$ and displaces $\mathrm{BH} 3$ proteins with an $\mathrm{IC}_{50}$ of $0.5 \mu \mathrm{M}$ [13]; subsequent derivatives of gossypol interact with a spectrum of anti-apoptotic BCL-2 family members with submicromolar affinities $[14,15]$. Gossypol treatment of primary lymphocytes taken from CLL patients induces caspase-independent apoptosis [16]. AT-101 ("apo-gossypol") is an enantiomer of gossypol (R(-)-gossypol) that binds BCL-2, BCL- $\mathrm{X}_{\mathrm{L}}$, and MCL-1 and

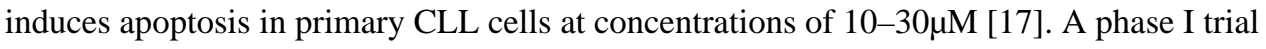
of AT-101 monotherapy in patients with relapsed/refractory CLL identified transaminitis as a major toxicity, with preliminary results indicating limited efficacy [18]. A subsequent phase II study of AT-101 in combination with rituximab for relapsed/refractory CLL treatment reported a preliminary overall response rate (ORR) of $44 \%$ with no complete responses [19].

\section{Obatoclax}

Obatoclax (GX15-070) is a small molecule identified in a screen for compounds that specifically disrupt protein-protein interactions between BCL-2 family members [20]. It is considered a "pan-BCL-2" family inhibitor, with the ability to bind BCL-2, BCL- $\mathrm{X}_{\mathrm{L}}$, BCL-

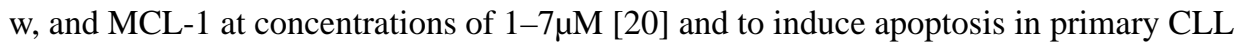
cells in vitro [21]. Obatoclax-induced cell death, however, is only partially abrogated by deletion of BAX and BAK, suggesting that the drug likely also causes cytotoxicity through mechanisms other than MOMP and apoptosis [22]. Phase I studies of obatoclax in CLL patients demonstrated significant toxicities with limited efficacy. Neurologic symptoms such as somnolence, ataxia, and confusion were found to be dose-limiting [23]. Only 1/26 patients $(4 \%)$ in the phase I trial of obtatoclax monotherapy experienced a partial response [23]; a phase I trial of obatoclax in combination with fludarabine and rituximab for relapsed/ refractory CLL had a partial response rate of 54\% [24]. The toxicities of obatoclax, along with its limited efficacy compared to navitoclax and venetoclax, ultimately limited its development as a therapeutic agent for CLL.

In summary, despite some promising pre-clinical data, multiple early attempts to inhibit BCL-2 family members in patients were largely unsuccessful. Given the compelling biology 
of BCL-2 dependence in the pathophysiology of CLL, this lack of early success did not dissuade investigators from pursuing BCL-2 as a therapeutic target.

\section{More Recent Attempts at Targeting BCL-2 in the Clinic}

\section{Navitoclax}

A breakthrough in the development of BCL-2 inhibitors occurred through a screen for small molecules that block the hydrophobic BH3-binding domain of BCL- $\mathrm{X}_{\mathrm{L}}$ [25], which eventually identified ABT-737, which binds to BCL-2, BCL- $\mathrm{X}_{\mathrm{L}}$, and BCL-w with high affinity $\left(\mathrm{K}_{\mathrm{i}} \leq 1 \mathrm{nM}\right)$. This binding disrupts their interactions with pro-apoptotic BH3-only family members, which are then free to bind to BAX/BAK, leading to oligomerization and MOMP. Navitoclax (ABT-263) is a second generation, structurally related molecule that is orally available and has more favorable pharmacokinetics [26]. It has an oral bioavailability of 20-50\% and a half-life of 8.9 hours [26], making it suitable for once-daily dosing. Its specificity mirrors that of ABT-737, with a $\mathrm{K}_{\mathrm{i}}$ of $\triangle \mathrm{nM}$ against BCL-2, BCL- $\mathrm{X}_{\mathrm{L}}$, and BCL$\mathrm{w}$, and a $\mathrm{K}_{\mathrm{i}}$ of 550nM against MCL-1 [26].

Promising pre-clinical findings [26, 27] led to the development of clinical trials in lymphoid malignancies. In a phase I trial of navitoclax in 55 patients with a variety of lymphoid malignancies, the subset of 20 patients with CLL/SLL were found to be particularly responsive to the drug, with a median progression free survival (PFS) of 246 days [28]. Subsequently, a phase I study of navitoclax restricted to patients with relapsed/refractory CLL was undertaken [29]. Nine out of 29 patients (31\%) achieved a partial response, and $90 \%$ of patients had at least a $50 \%$ reduction in their peripheral blood lymphocyte count. Notably, responses were fairly durable, with a median PFS of 25 months in a heavily pretreated group of patients. An open-label, randomized phase II study compared navitoclax plus rituximab to rituximab alone in previously untreated CLL. The addition of rituximab to 12 weeks of navitoclax led to an ORR of 55\%, compared to $35 \%$ for patients treated with rituximab monotherapy. The combination of rituximab with navitoclax given until time of progression further increased the ORR to $70 \%$ [30].

The dose limiting toxicity of navitoclax was a dose-dependent reduction in platelet count, with grade 33 thrombocytopenia (platelet count $<50,000$ ) occurring in $28 \%$ of patients in the phase I CLL study [29] and 26\% of patients in a phase II study [30]. This was attributed to BCL- $\mathrm{X}_{\mathrm{L}}$ inhibition in platelets [31], and prompted a drive to identify an inhibitor that retained activity against BCL-2 but lacked activity against BCL- $\mathrm{X}_{\mathrm{L}}$.

\section{Venetoclax}

Venetoclax (ABT-199/GDC-0199) is the result of reverse engineering of navitoclax to increase BCL-2 selectivity [32] (Figure 1). Accordingly, venetoclax has subnanomolar affinity for BCL-2 $\left(\mathrm{K}_{\mathrm{i}}<0.010 \mathrm{nM}\right)$, but significantly weaker binding to BCL- $\mathrm{X}_{\mathrm{L}}\left(\mathrm{K}_{\mathrm{i}}=48 \mathrm{nM}\right)$, BCL-w $\left(\mathrm{K}_{\mathrm{i}}=245 \mathrm{nM}\right)$, and MCL-1 $\left(\mathrm{K}_{\mathrm{i}}>444 \mathrm{nM}\right)$ [32]. Venetoclax has adequate oral bioavailability and an estimated half-life of 26 hours [33, 34]. Consistent with the known BCL-2 dependence of CLL cells, venetoclax treatment in vitro induced apoptosis in primary CLL cells, with a remarkable $\mathrm{EC}_{50}$ of $3 \mathrm{nM}$ [32]. 
Venetoclax first-in-human study-A first-in-human phase I study of venetoclax was initiated in 2011, with initial dosing ranging from 100-200mg. Of the first three patients treated, the two with peripheral lymphocytosis experienced a rapid reduction in the white blood cell count within 8 hours of the first dose. All three patients developed laboratory evidence of tumor lysis syndrome (TLS) without clinical sequelae, and all three patients were able to safely resume dosing after resolution of TLS [32].

Due to the TLS observed in these first 3 patients, the dosing regimen on this study was modified to include a lower starting dose of $50 \mathrm{mg}$ as well as a weekly intrapatient dose ramp-up to a target dose for each dosing cohort, up to a maximum dose of $1200 \mathrm{mg}$ daily. The multicenter, open-label phase 1 study ultimately enrolled 116 patients with relapsed or refractory CLL or SLL [35]. Patients received daily venetoclax until disease progression or unacceptable toxicity. Despite the initial revision to start at a lower dose of $50 \mathrm{mg}$, there were 2 clinically significant cases of TLS (one case resulting in the need for hemodialysis and one fatality). This led to further revision of the dosing scheme, with venetoclax beginning at 20mg followed by weekly intrapatient dose ramp-up over 5 weeks to $50 \mathrm{mg}$, $100 \mathrm{mg}, 200 \mathrm{mg}$, and finally $400 \mathrm{mg}$ per day. Patients were monitored closely for TLS, including hospitalization for those with a high burden of disease, and supportive measures such as intravenous hydration and uric acid lowering agents were mandated. Notably, none of the patients treated with this most recent dosing regimen experienced clinical TLS. Neutropenia (in $45 \%$ of patients, with $41 \%$ grade $\geq 3$ ) and gastrointestinal side effects (diarrhea in $52 \%$ of patients, with $2 \%$ grade 33 ; nausea in $47 \%$ of patients, with $2 \%$ grade 23) were common adverse events. The drug was highly efficacious as monotherapy, even in high risk patients such as those with del(17p) and those refractory to fludarabine-based chemoimmunotherapy. The ORR in all patients, including both the dose-escalation cohort and the expansion cohort, was $79 \%$. In the subgroup of patients with a $17 \mathrm{p}$ deletion, the ORR remained high at $71 \%$, and in the subgroup of patients refractory to fludarabine, the ORR was $79 \%$. However, while the ORR in the high-risk 17p-deleted subgroup of patients was similar to the overall cohort, this cohort did have a significantly shorter PFS than the non-17p-deleted cohort. Complete responses (CRs) by IW-CLL criteria [36] were seen in $20 \%$ of patients (with comparable CR rates in the high-risk subgroups), and 5\% of patients achieved minimal residual disease (MRD) negativity. A report with longer follow-up on the cohort of patients who received the recommended phase II dose of $400 \mathrm{mg} /$ day was notable for an ORR of $81 \%$ (CR rate of $16 \%$ ) and also highlighted the durability of responses, with an estimated 24 month PFS of $62 \%$ [37].

Venetoclax in del(17p) CLL-The success of venetoclax monotherapy in the 17p-deleted population was later confirmed in a landmark phase II, single-arm, open-label, multicenter study in 107 subjects receiving venetoclax 400mg daily for relapsed, refractory, 17p-deleted CLL [38]. Subjects continued venetoclax until disease progression or unacceptable toxicity. To reduce the risk of TLS, a stepwise ramp-up dosing strategy was again used. The ORR was $79 \%$ with an $8 \% \mathrm{CR}$ rate. With a median duration of follow-up of only 12 months, it is possible that the $\mathrm{CR}$ rate may increase over time based on experience from the phase I study. In April 2016, venetoclax received FDA approval for the treatment of patients with 17pdeleted CLL who have relapsed after or are refractory to at least one prior line of therapy. 
Venetoclax for patients who progress on BCR pathway inhibitors-Venetoclax has a distinct mechanism of action from the $\mathrm{B}$ cell receptor (BCR) pathway inhibitors such as the BTK inhibitor ibrutinib and the PI3K- $\delta$ inhibitor idelalisib. Thus, one would hypothesize that venetoclax may be effective in patients who have progressed on these kinase inhibitors. This hypothesis is being tested in a phase II study of venetoclax in patients who have CLL that is relapsed after, or refractory to, ibrutinib or idelalisib. Venetoclax monotherapy is dosed as per the latest schedule from the prior trials, and it is continued until disease progression. Early results from 64 patients have been presented in abstract form [39], with an ORR of $70 \%$ in patients who progressed on ibrutinib and $48 \%$ in patients who progressed on idelalisib. Several CRs were seen despite the short follow-up.

Venetoclax combination studies-While venetoclax clearly has efficacy in CLL as monotherapy, there is also good justification for using it as a component of combination therapy. Its mechanism of action and toxicity profile are different from that of other approved agents for CLL, and combination therapy may be able to achieve deeper, more durable responses than venetoclax alone.

Venetoclax may synergistically enhance antibody dependent cytotoxicity [40], and thus some initial studies have explored the combination with anti-CD20 monoclonal antibodies. In results from a phase Ib, open-label, multicenter study of venetoclax plus rituximab in 49 patients, the ORR was $86 \%$ [41], with $51 \%$ of patients achieving CR or CRi after a median 28 months of follow-up, exceeding the CR rates seen with monotherapy (although patients on this combination were less heavily pre-treated than those on the monotherapy studies). Eighty percent of patients with CR/CRi had no minimal residual disease (MRD) by $\geq 4$ color flow cytometry on bone marrow biopsy. The toxicity profile, including neutropenia (55\%), diarrhea (57\%), and nausea (51\%), was similar to that seen with venetoclax alone. There was one fatal TLS event prior to a protocol modification specifying parameters for TLS risk management and prophylaxis. Based on the results of this trial, venetoclax was given breakthrough therapy designation by the FDA in combination with rituximab for the treatment of relapsed/refractory CLL. Venetoclax, bendamustine, and rituximab triplet combination therapy is being investigated in a phase I study in both relapsed/refractory and untreated CLL; preliminary results showed an ORR of $96 \%$ in 30 patients with relapsed/ refractory disease [42]. In similar fashion, venetoclax has been combined with obinutuzumab in a currently ongoing phase Ib study in both relapsed/refractory as well as untreated CLL [43]. In the 17 enrolled patients with relapsed/refractory disease, the preliminary ORR is $100 \%$, with a CR/CRi rate of $23.5 \%$.

These early phase clinical trial findings will need to be confirmed in large, randomized trials. The MURANO trial is a phase III study randomizing patients with relapsed/refractory CLL to either six cycles of venetoclax/rituximab therapy (followed by venetoclax maintenance therapy for up to 24 months) or six cycles of bendamustine/rituximab. It has completed enrollment, but results have not yet been reported. The CLL14 trial, a phase III study of venetoclax/obinutuzumab versus chlorambucil/obinutuzumab in previously untreated older patients with CLL, is also now fully accrued, and if positive could help define a new standard of care for these patients [44]. 
Additional trials have recently launched to study an all-oral regimen of venetoclax in combination with ibrutinib. Using BH3 profiling, ibrutinib has been shown to enhance ex vivo CLL cell sensitivity to venetoclax, in part by upregulating the proapoptotic BIM protein [45] and decreasing expression of BCL-X $\mathrm{L}_{\mathrm{L}}$ and MCL-1 [46]. A non-randomized, phase II study of ibrutinib in combination with venetoclax (NCT02756897) is currently enrolling patients, and two phase II trials have also recently launched to examine the three drug combination of ibrutinib, venetoclax, and obinutuzumab in previously untreated patients with 17p-deleted CLL (NCT02758665) and in previously untreated or relapsed/refractory patients in any cytogenetic risk group (NCT02427451). A summary of the trials of venetoclax in CLL can be found in Table 1.

\section{Safely administering venetoclax}

The numerous clinical trials investigating the role of venetoclax in the treatment of CLL have provided initial insight into its toxicity profile. The dose-dependent thrombocytopenia seen with navitoclax has not been observed with venetoclax. TLS has been the most concerning adverse event observed, and optimal TLS prophylaxis requires both careful assessment and management of risk. TLS risk stratification requires assessment of disease burden in the peripheral blood and lymph nodes. Low risk patients have no lymph nodes $\geq$ $5 \mathrm{~cm}$ in diameter and have an absolute lymphocyte count $<25 \times 10^{9}$ cells $/ \mathrm{L}$, high risk patients meet both of these criteria (or have any lymph node $\geq 10 \mathrm{~cm}$ in size), and medium risk patients meet only one of these criteria. Patients with pre-existing renal impairment should also be considered high risk. Regardless of risk category, patients initiating venetoclax should receive allopurinol, aggressive oral or intravenous hydration, and laboratory monitoring for TLS. Additionally, high risk patients should receive their initial doses of venetoclax in an inpatient setting, with prophylactic rasburicase and even more intensive laboratory monitoring. Utilizing this approach, the rate of laboratory TLS was $4 \%$ in a recent integrated safety analysis of nearly 300 patients treated on three clinical trials with venetoclax monotherapy [48]. All of these events occurred during the ramp-up phase of venetoclax dosing, and none resulted in clinical sequelae.

One of the most frequent adverse events with venetoclax monotherapy is neutropenia, with an incidence of $38 \%$ (and $36 \%$ for grade $\geq 3$ neutropenia [absolute neutrophil count $<1,000$ cells/ $\mu \mathrm{L}]$ ) [48]. The presumed mechanism is due to on-target BCL-2 inhibition in neutrophil precursors. The incidence of febrile neutropenia has been low at approximately 5\% [48] and can be managed safely with growth factor support given concomitantly with venetoclax. Less commonly, venetoclax dose interruption or dose reduction may be required. Outside of effects on neutrophils, the impact of venetoclax on the immune system seems modest, with negligible impact on T-cells, NK cells, or immunoglobulin levels [37]. Gastrointestinal side effects -- such as nausea, vomiting, constipation, and diarrhea -- impact approximately half of all patients receiving the drug, but are generally low grade and transient.

\section{Unanswered Questions with BCL-2 Inhibition}

The clinical experience with BCL-2 inhibitors, particularly venetoclax, has prompted some interesting yet currently unanswered questions. For example, recent data have suggested that 
patients who achieve MRD-negativity with chemoimmunotherapy, either with a CR or a PR, have superior PFS and overall survival (OS) compared to patients whose best response is MRD-positive, even if they are otherwise in a CR [49]. One important question is whether achieving MRD-negativity with venetoclax is also predictive of a longer PFS and OS. Compared to BTK inhibitors, which have a CR/CRi rate of 0-7\% in relapsed/refractory patients [50,51], venetoclax appears to be superior at achieving CRs, in many cases with MRD-negativity. If achievement of MRD-negativity with venetoclax can be validated as predictive of a long-term disease-free state, both patient care and future trial design would be greatly impacted. This would suggest that venetoclax would be an excellent backbone upon which future front-line combination regimens can be built.

Closely related to the issue of achieving MRD-negativity is the unresolved question regarding the optimal dosing duration for venetoclax. Should venetoclax be continued until progression, or can it be stopped after a fixed period of time (particularly if the patient has achieved MRD-negativity)? The phase Ib trial of venetoclax plus rituximab gives subjects the option of discontinuing therapy after achieving a CR/CRi (a subsequent amendment also required MRD negativity in the bone marrow at time of treatment cessation). Thirteen subjects discontinued therapy. Only two of the 13 (both of whom were MRD positive) had relapsed after a median of 9.7 months off all therapy. Both of these subjects then restarted venetoclax and achieved a partial response [41]. These preliminary data suggest that venetoclax can be discontinued in some patients and retreatment can be effective, but larger numbers are needed to confirm this strategy. Additional studies of venetoclax with a fixed duration of therapy will provide further insight into this question.

Another key issue with venetoclax is whether predictive biomarkers for response can be identified. A recent study of samples taken from venetoclax-treated patients showed that neither TP53 status nor in vitro sensitivity of CLL cells to the drug predicted depth of response to venetoclax in patients. However, $\mathrm{BH} 3$ profiling was able to significantly identify the subset of patients who had a greater reduction in CLL bone marrow infiltrate [52]. Further validation of these initial findings will require systematic evaluation of a larger cohort of patients.

Another critical issue is elucidating mechanisms of resistance to BCL-2 inhibition. Preclinically, there is evidence to suggest that upregulation of alternative anti-apoptotic BCL-2 family members may lead to BCL-2 inhibitor resistance. For example, one group used stromal co-culture of CLL cells to mimic the lymph node microenvironment, and observed increased expression of BCL- $\mathrm{X}_{\mathrm{L}}$ and BCL2A1, which correlated with a change in the $\mathrm{EC}_{50}$ of ABT-737 from $7 \mathrm{nM}$ to $>10 \mu \mathrm{M}$ [53]. Another group treated 7 lymphoma and leukemia cell lines in long term culture with steadily increasing concentrations of venetoclax, eventually generating clones that had 100-fold less sensitivity to the drug [54]. The clones displayed reductions in the proapoptotic proteins BAX, BIM, and NOXA and increases in BCL- $\mathrm{X}_{\mathrm{L}}$ and MCL-1. These findings were recapitulated by a second group which demonstrated upregulation of BCL- $\mathrm{X}_{\mathrm{L}}$ and MCL-1 in lymphoma cell lines that became resistant to venetoclax after chronic exposure [55]. Mutations in BCL-2 that block venetoclax binding, and an inactivating mutation in BAX that blocks the protein's ability to attach to the outer mitochondrial membrane, have also been described in cell line models of 
acquired venetoclax resistance [56]. Clinically, a significant number of patients who have progressed on venetoclax have done so through Richter's transformation, which accounted for $44 \%$ of progression events in the phase I first-in-human study of venetoclax monotherapy [35]. As this phenomenon has also been observed in CLL patients treated with other novel agents such as ibrutinib, these transformation events may reflect the fact that novel agents allow patients with genetically unstable (e.g. TP53 deleted) disease to live longer, providing more time for transformation to occur, rather than inducing transformation directly.

\section{Conclusions}

The development of BCL-2 inhibitors for use in CLL represents a triumph of rational scientific investigation. While initial attempts at BCL-2 family inhibition did not achieve the desired specificity and efficacy, rational drug design eventually yielded a viable approach with $\mathrm{BH} 3$ mimetics. And while the early experience with navitoclax was hampered by the unanticipated development of thrombocytopenia, this problem was overcome with the BCL-2 specific inhibitor venetoclax, an agent so effective in CLL that its most serious toxicity is TLS due to rapid tumor cell killing. The task now turns to clinical investigators to identify the best way to incorporate venetoclax into the expanding arsenal of drugs for the treatment of CLL. Future studies in CLL will focus on venetoclax combination therapies, timing and duration of venetoclax dosing, predictors of response, and mechanisms of resistance. While many unanswered questions yet remain, the future of BCL-2 inhibition in CLL treatment is bright.

\section{Bibliography}

1. Delbridge AR, Grabow S, Strasser A, Vaux DL. Thirty years of BCL-2: translating cell death discoveries into novel cancer therapies. Nat Rev Cancer. 2016; 16(2):99-109. [PubMed: 26822577]

2. Pugazhenthi S, Nesterova A, Sable C, et al. Akt/protein kinase B up-regulates Bcl-2 expression through cAMP-response element-binding protein. J Biol Chem. 2000; 275(15):10761-10766. [PubMed: 10753867]

3. Nakano K, Vousden KH. PUMA, a novel proapoptotic gene, is induced by p53. Mol Cell. 2001; 7(3):683-694. [PubMed: 11463392]

4. Leber B, Lin J, Andrews DW. Embedded together: the life and death consequences of interaction of the Bcl-2 family with membranes. Apoptosis. 2007; 12(5):897-911. [PubMed: 17453159]

5. Certo M, Del Gaizo Moore V, Nishino M, et al. Mitochondria primed by death signals determine cellular addiction to antiapoptotic BCL-2 family members. Cancer Cell. 2006; 9(5):351-365. [PubMed: 16697956]

6. Del Gaizo Moore V, Letai A. BH3 profiling--measuring integrated function of the mitochondrial apoptotic pathway to predict cell fate decisions. Cancer Lett. 2013; 332(2):202-205. [PubMed: 22230093]

7. Del Gaizo Moore V, Brown JR, Certo M, Love TM, Novina CD, Letai A. Chronic lymphocytic leukemia requires BCL2 to sequester prodeath BIM, explaining sensitivity to BCL2 antagonist ABT-737. J Clin Invest. 2007; 117(1):112-121. [PubMed: 17200714]

8. Davids MS. Boldly Targeting Kinases without mutations. Blood. 2014; 123(8):1119-1121. [PubMed: 24558189]

9. Schlagbauer-Wadl H, Klosner G, Heere-Ress E, et al. Bcl-2 antisense oligonucleotides (G3139) inhibit Merkel cell carcinoma growth in SCID mice. J Invest Dermatol. 2000; 114(4):725-730. [PubMed: 10733680] 
10. Ramanarayanan J, Hernandez-Ilizaliturri FJ, Chanan-Khan A, Czuczman MS. Pro-apoptotic therapy with the oligonucleotide Genasense (oblimersen sodium) targeting Bcl-2 protein expression enhances the biological anti-tumour activity of rituximab. Br J Haematol. 2004; 127(5): 519-530. [PubMed: 15566355]

11. O’Brien SM, Cunningham CC, Golenkov AK, Turkina AG, Novick SC, Rai KR. Phase I to II multicenter study of oblimersen sodium, a Bcl-2 antisense oligonucleotide, in patients with advanced chronic lymphocytic leukemia. J Clin Oncol. 2005; 23(30):7697-7702. [PubMed: 16186597]

12. O'Brien S, Moore JO, Boyd TE, et al. 5-year survival in patients with relapsed or refractory chronic lymphocytic leukemia in a randomized, phase III trial of fludarabine plus cyclophosphamide with or without oblimersen. J Clin Oncol. 2009; 27(31):5208-5212. [PubMed: 19738118]

13. Kitada S, Leone M, Sareth S, Zhai D, Reed JC, Pellecchia M. Discovery, characterization, and structure-activity relationships studies of proapoptotic polyphenols targeting B-cell lymphocyte/ leukemia-2 proteins. J Med Chem. 2003; 46(20):4259-4264. [PubMed: 13678404]

14. Wei J, Kitada S, Rega MF, et al. Apogossypol derivatives as pan-active inhibitors of antiapoptotic B-cell lymphoma/leukemia-2 (Bcl-2) family proteins. J Med Chem. 2009; 52(14):4511-4523. [PubMed: 19555126]

15. Becattini B, Kitada S, Leone M, et al. Rational design and real time, in-cell detection of the proapoptotic activity of a novel compound targeting Bcl-X(L). Chem Biol. 2004; 11(3):389-395. [PubMed: 15123268]

16. Balakrishnan K, Wierda WG, Keating MJ, Gandhi V. Gossypol, a BH3 mimetic, induces apoptosis in chronic lymphocytic leukemia cells. Blood. 2008; 112(5):1971-1980. [PubMed: 18566329]

17. Balakrishnan K, Burger JA, Wierda WG, Gandhi V. AT-101 induces apoptosis in CLL B cells and overcomes stromal cell-mediated Mcl-1 induction and drug resistance. Blood. 2009; 113(1):149153. [PubMed: 18836097]

18. James DF, Castro JE, Loria O, Prada CE, Aguillon RA, Kipps TJ. AT-101, a small molecule Bcl-2 antagonist, in treatment naive CLL patients (pts) with high risk features; Preliminary results from an ongoing phase I trial. ASCO Meeting Abstracts. 2006; 24(18_suppl):6605.

19. Castro JE, Loria OJ, Aguillon RA, et al. A Phase II, Open Label Study of AT-101 in Combination with Rituximab in Patients with Relapsed or Refractory Chronic Lymphocytic Leukemia. Evaluation of Two Dose Regimens Blood. 2007; 110(11):3119-3119.

20. Nguyen M, Marcellus RC, Roulston A, et al. Small molecule obatoclax (GX15-070) antagonizes MCL-1 and overcomes MCL-1-mediated resistance to apoptosis. Proc Natl Acad Sci U S A. 2007; 104(49):19512-19517. [PubMed: 18040043]

21. Perez-Galan P, Roue G, Lopez-Guerra M, et al. BCL-2 phosphorylation modulates sensitivity to the BH3 mimetic GX15-070 (Obatoclax) and reduces its synergistic interaction with bortezomib in chronic lymphocytic leukemia cells. Leukemia. 2008; 22(9):1712-1720. [PubMed: 18596739]

22. Konopleva M, Watt J, Contractor R, et al. Mechanisms of antileukemic activity of the novel Bcl-2 homology domain-3 mimetic GX15-070 (obatoclax). Cancer Res. 2008; 68(9):3413-3420. [PubMed: 18451169]

23. O'Brien SM, Claxton DF, Crump M, et al. Phase I study of obatoclax mesylate (GX15-070), a small molecule pan-Bcl-2 family antagonist, in patients with advanced chronic lymphocytic leukemia. Blood. 2009; 113(2):299-305. [PubMed: 18931344]

24. Brown JR, Tesar B, Yu L, et al. Obatoclax in combination with fludarabine and rituximab is welltolerated and shows promising clinical activity in relapsed chronic lymphocytic leukemia. Leuk Lymphoma. 2015; 56(12):3336-3342. [PubMed: 25971907]

25. Oltersdorf T, Elmore SW, Shoemaker AR, et al. An inhibitor of Bcl-2 family proteins induces regression of solid tumours. Nature. 2005; 435(7042):677-681. [PubMed: 15902208]

26. Tse C, Shoemaker AR, Adickes J, et al. ABT-263: a potent and orally bioavailable Bcl-2 family inhibitor. Cancer Res. 2008; 68(9):3421-3428. [PubMed: 18451170]

27. Vogler M, Furdas SD, Jung M, Kuwana T, Dyer MJ, Cohen GM. Diminished sensitivity of chronic lymphocytic leukemia cells to ABT-737 and ABT-263 due to albumin binding in blood. Clin Cancer Res. 2010; 16(16):4217-4225. [PubMed: 20601444] 
28. Wilson WH, O'Connor OA, Czuczman MS, et al. Navitoclax, a targeted high-affinity inhibitor of BCL-2, in lymphoid malignancies: a phase 1 dose-escalation study of safety, pharmacokinetics, pharmacodynamics, and antitumour activity. Lancet Oncol. 2010; 11(12):1149-1159. [PubMed: 21094089]

29. Roberts AW, Seymour JF, Brown JR, et al. Substantial susceptibility of chronic lymphocytic leukemia to BCL2 inhibition: results of a phase I study of navitoclax in patients with relapsed or refractory disease. J Clin Oncol. 2012; 30(5):488-496. [PubMed: 22184378]

30. Kipps TJ, Eradat H, Grosicki S, et al. A phase 2 study of the BH3 mimetic BCL2 inhibitor navitoclax (ABT-263) with or without rituximab, in previously untreated B-cell chronic lymphocytic leukemia. Leuk Lymphoma. 2015; 56(10):2826-2833. [PubMed: 25797560]

31. Schoenwaelder SM, Jarman KE, Gardiner EE, et al. Bcl-xL-inhibitory BH3 mimetics can induce a transient thrombocytopathy that undermines the hemostatic function of platelets. Blood. 2011; 118(6):1663-1674. [PubMed: 21673344]

32•**. Souers AJ, Leverson JD, Boghaert ER, et al. ABT-199, a potent and selective BCL-2 inhibitor, achieves antitumor activity while sparing platelets. Nat Med. 2013; 19(2):202-208. This study describes the rational development of venetoclax as a BCL-2 specific inhibitor, and also includes analysis of the first three patients dosed with venetoclax. [PubMed: 23291630]

33. Salem AH, Agarwal S, Dunbar M, et al. Effect of Low and High Fat Meals on the Pharmacokinetics of Venetoclax, a Selective First-in-Class Bcl-2 Inhibitor. J Clin Pharmacol. 2016

34. Jones AK, Freise KJ, Agarwal SK, Humerickhouse RA, Wong SL, Salem AH. Clinical Predictors of Venetoclax Pharmacokinetics in Chronic Lymphocytic Leukemia and Non-Hodgkin's Lymphoma Patients: a Pooled Population Pharmacokinetic Analysis. AAPS J. 2016

35•**. Roberts AW, Davids MS, Pagel JM, et al. Targeting BCL2 with Venetoclax in Relapsed Chronic Lymphocytic Leukemia. N Engl J Med. 2016; 374(4):311-322. This phase 1, first-inhuman study established venetoclax monotherapy as an efficacious approach for the treatment of relapsed, refractory CLL. [PubMed: 26639348]

36. Hallek M, Cheson BD, Catovsky D, et al. Guidelines for the diagnosis and treatment of chronic lymphocytic leukemia: a report from the International Workshop on Chronic Lymphocytic Leukemia updating the National Cancer Institute-Working Group 1996 guidelines. Blood. 2008; 111(12):5446-5456. [PubMed: 18216293]

37. Davids MS, Roberts AW, Seymour JF, et al. Safety, efficacy and immune effects of venetoclax 400 mg daily in patients with relapsed chronic lymphocytic leukemia (CLL). ASCO Meeting Abstracts. 2016; 34(15_suppl):7527.

38* *. Stilgenbauer S, Eichhorst B, Schetelig J, et al. Venetoclax in relapsed or refractory chronic lymphocytic leukaemia with 17p deletion: a multicentre, open-label, phase 2 study. Lancet Oncol. 2016; 17(6):768-778. This study demonstrated that venetoclax is highly efficacious even in the high-risk subgroup of patients with relapsed, refractory 17p-deleted CLL. [PubMed: 27178240]

39. Jones JA, Wierda WG, Choi MY, et al. Venetoclax activity in CLL patients who have relapsed after or are refractory to ibrutinib or idelalisib. ASCO Meeting Abstracts. 2016; 34(15_suppl):7519.

40. Sampath D, Herter S, Ingalla E, et al. Combination of the Glycoengineered Type II CD20 Antibody Obinutuzumab with the Novel BCL-2 Selective Inhibitor Venetoclax Induces Robust Cell Death in NHL Models and CLL Patient Samples. Haematologica. 2016; 101(s1):1-881. [PubMed: 26721798]

41•**. Seymour JF, Ma S, Brander DM, et al. Venetoclax plus rituximab in relapsed or refractory chronic lymphocytic leukaemia: a phase Ib study. Lancet Oncology. 2017; 18(2):230-240. This is the first published study to evaluate venetoclax as part of a combination regimen, demonstrating that deep and durable responses can be achieved when venetoclax is used in combination with rituximab. [PubMed: 28089635]

42. Stilgenbauer S, Morschhauser F, Wendtner C-M, et al. Phase Ib Study (GO28440) of venetoclax with bendamustine/rituximab or bendamustine/obinutuzumab in patients with relapsed/refractory or previously untreated chronic lymphocytic leukemia. Blood. 2016; 128(22):4393.

43. Flinn IW, Brunvand M, Choi MY, et al. Safety and Efficacy of a Combination of Venetoclax (GDC-0199/ABT-199) and Obinutuzumab in Patients with Relapsed/Refractory or Previously 
Untreated Chronic Lymphocytic Leukemia - Results from a Phase 1b Study (GP28331). Blood. 2015; 126(23):494-494. [PubMed: 25987658]

44. Fischer K, Fink A-M, Bishop H, et al. Results of the Safety Run-in Phase of CLL14 (BO25323): A Prospective, Open-Label, Multicenter Randomized Phase III Trial to Compare the Efficacy and Safety of Obinutuzumab and Venetoclax (GDC-0199/ABT-199) with Obinutuzumab and Chlorambucil in Patients w.... Blood. 2015; 126(23):496-496.

45. Deng J, Isik E, Fernandes SM, Brown JR, Letai A, Davids MS. Ibrutinib Therapy Increases BCL-2 Dependence and Enhances Sensitivity to Venetoclax in CLL. Blood. 2015; 126(23):490-490.

46. Cervantes-Gomez F, Lamothe B, Woyach JA, et al. Pharmacological and Protein Profiling Suggests Venetoclax (ABT-199) as Optimal Partner with Ibrutinib in Chronic Lymphocytic Leukemia. Clin Cancer Res. 2015; 21(16):3705-3715. [PubMed: 25829398]

47. Jones JA, Woyach J, Awan FT, et al. Phase $1 \mathrm{~b}$ results of a phase $1 \mathrm{~b} / 2$ Study of obinutuzmab, ibrutinib, and venetoclax in relapsed/refractory chronic lymphocytic leukemia (CLL). Blood. 2016; 128(22):639.

48. Davids MS, Gerecitano JF, Potluri J, et al. Integrated safety analysis of venetoclax monotherapy in chronic lymphocytic leukemia (CLL). ASCO Meeting Abstracts. 2016; 34(15_suppl):7528.

49. Kovacs G, Robrecht S, Fink AM, et al. Minimal Residual Disease Assessment Improves Prediction of Outcome in Patients With Chronic Lymphocytic Leukemia (CLL) Who Achieve Partial Response: Comprehensive Analysis of Two Phase III Studies of the German CLL Study Group. J Clin Oncol. 2016

50. Byrd JC, Furman RR, Coutre SE, et al. Three-year follow-up of treatment-naive and previously treated patients with CLL and SLL receiving single-agent ibrutinib. Blood. 2015; 125(16):24972506. [PubMed: 25700432]

51. Byrd JC, Harrington B, O’Brien S, et al. Acalabrutinib (ACP-196) in Relapsed Chronic Lymphocytic Leukemia. N Engl J Med. 2016; 374(4):323-332. [PubMed: 26641137]

52**. Anderson MA, Deng J, Seymour JF, et al. The BCL2 selective inhibitor venetoclax induces rapid onset apoptosis of CLL cells in patients via a TP53-independent mechanism. Blood. 2016; 127(25):3215-3224. This study demonstrated that venetoclax is effective at killing both TP53wildtype and TP53-mutant CLL cells to similar degrees, and also found that $\mathrm{BH} 3$ profiling may be able to predict depth of response to venetoclax in CLL patients. [PubMed: 27069256]

53. Vogler M, Butterworth M, Majid A, et al. Concurrent up-regulation of BCL-XL and BCL2A1 induces approximately 1000-fold resistance to ABT-737 in chronic lymphocytic leukemia. Blood. 2009; 113(18):4403-4413. [PubMed: 19008458]

54. Tahir SK, Smith ML, Hessler P, Roberts-Rapp L, Leverson JD, Lam LT. Abstract B30: Mechanisms of resistance to ABT-199 in leukemia and lymphoma cell lines. American Association for Cancer Research. 2015; 21(4 Supplement):B30-B30.

55. Choudhary GS, Al-Harbi S, Mazumder S, et al. MCL-1 and BCL-xL-dependent resistance to the BCL-2 inhibitor ABT-199 can be overcome by preventing PI3K/AKT/mTOR activation in lymphoid malignancies. Cell Death Dis. 2015; 6:e1593. [PubMed: 25590803]

56. Fresquet V, Rieger M, Carolis C, Garcia-Barchino MJ, Martinez-Climent JA. Acquired mutations in BCL2 family proteins conferring resistance to the BH3 mimetic ABT-199 in lymphoma. Blood. 2014; 123(26):4111-4119. [PubMed: 24786774] 


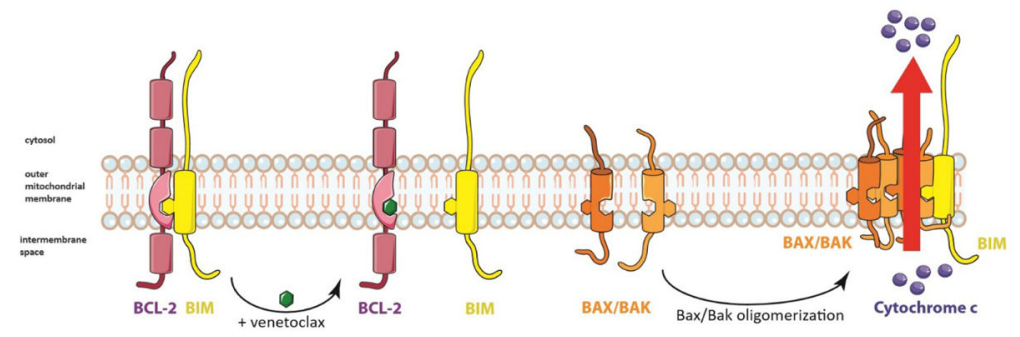

Figure 1. Mechanism of action of venetoclax

At baseline, BCL-2 and BIM exist in equilibrium on the outer mitochondrial membrane.

Venetoclax selectively antagonizes the interaction between the anti-apoptotic protein BCL-2 and the pro-apoptotic protein BIM, leading to BIM displacement from BCL-2 and recruitment of $\mathrm{BAX} / \mathrm{BAK}$ in active conformation to the mitochondrial membrane. $\mathrm{BAX} / \mathrm{BAK}$ homo-oligmerization lead to mitochondrial outer membrane permeabilization, cytochrome c release, and induction of caspase-mediated apoptosis. 


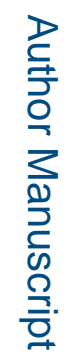

를

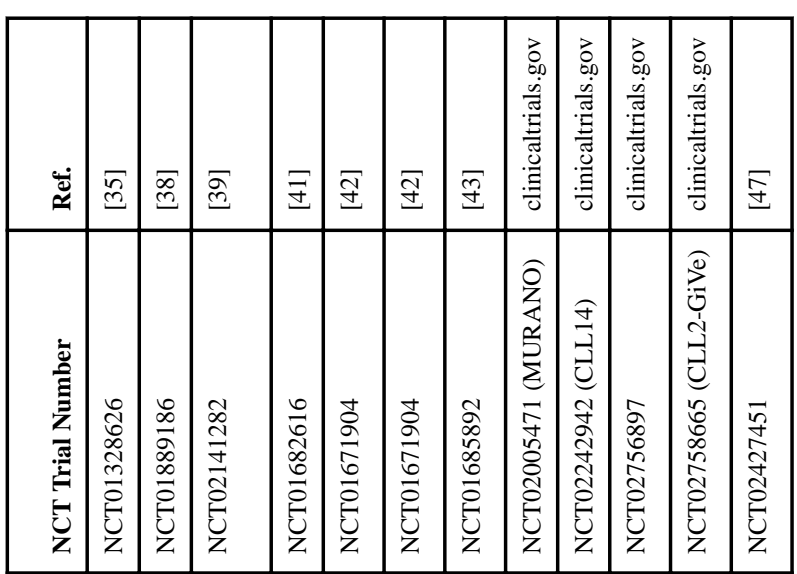

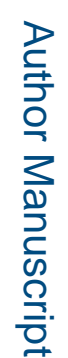

\begin{tabular}{l|l|l|l|l|l|l|l|l|l|}
\hline & & & & & & & & & \\
\hline
\end{tabular}

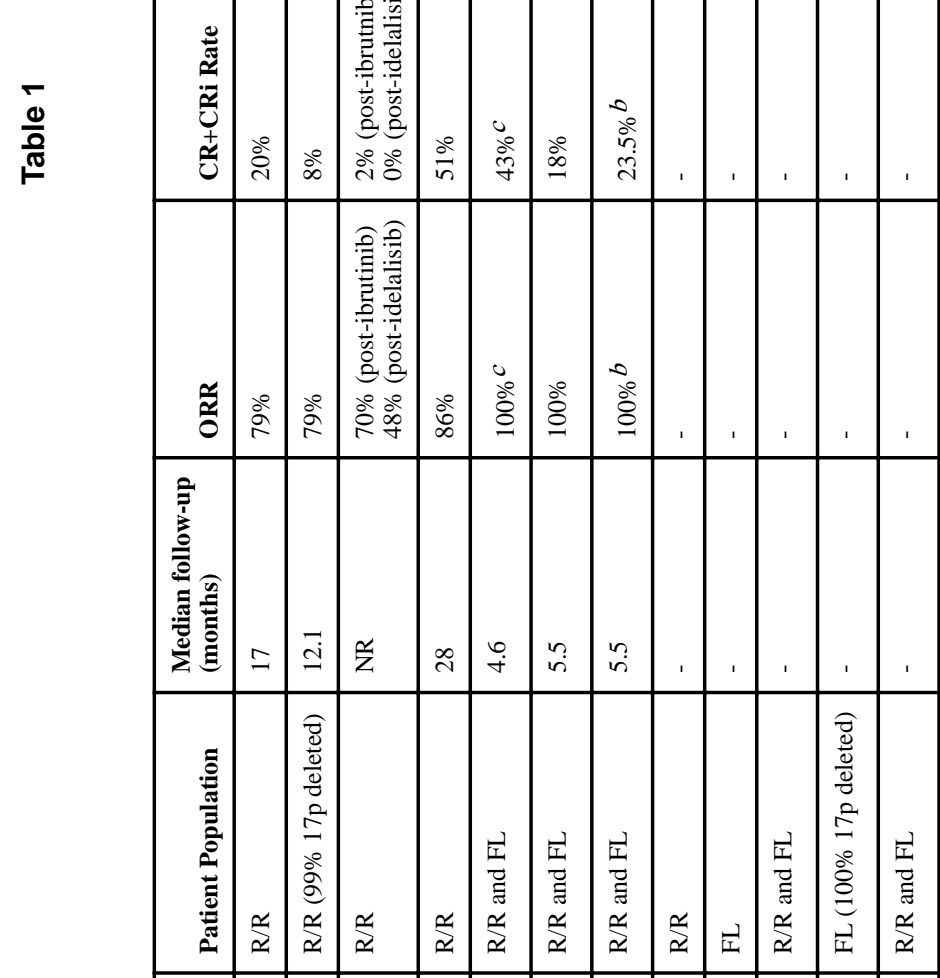

年

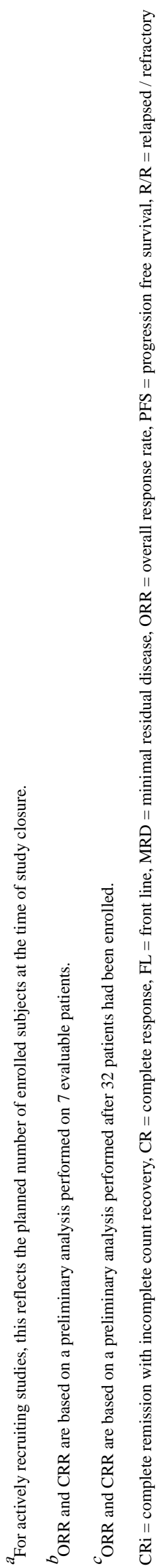

